\section{Jan Menke}

Received: 12 December 2008

Accepted: 4 April 2009

Published online: 16 May 2009

C) The Author(s) 2009.

This article is published with open access at Springerlink.com 2009

\section{J. Menke ( $\square)$}

Department of Diagnostic Radiology,

University Hospital,

Robert-Koch-Strasse 40,

37075 Goettingen, Germany

e-mail: Menke-J@T-Online.de

Tel.: +49-551-5053614

Fax: +49-551-5053614

\title{
Carotid MR angiography with traditional bolus timing: clinical observations and Fourier-based modelling of contrast kinetics
}

Abstract This study analyses the
relation between image quality and
contrast kinetics in bolus-timed
carotid magnetic resonance angiography
(MRA) and interprets the findings by Fourier-based numerical modelling. One hundred patients prone to carotid stenosis were studied using contrastenhanced carotid MRA with bolus timing. The carotid MRAs were timed to start relatively early without accounting for the injection time of the contrast medium. For interpretation different starting times were modelled, utilising the spectral information of the test bolus series. In the test bolus series the arterial time-to-peak showed a large $95 \%$ confidence interval of 12-27 s, indicating the need for individual MRA timing. All bolus-timed MRAs were of good diagnostic quality. The mean $( \pm \mathrm{SD})$ arterial contrast-to-noise ratio was $53.0( \pm 12.8)$ and thus high, and $95 \%$ of the MRAs showed a slight venous contamination of $11.8 \%$ or less (median 5.6\%). According to the Fourier-based modelling the central $k$-space may be acquired about $2 \mathrm{~s}$ before the arterial contrast peak. This results in carotid MRAs with sufficiently high arterial enhancement and little venous contamination. In conclusion, in bolus-timed carotid MRA a relatively short timing provides good arterial contrast with little venous contamination, which can be explained by Fourier-based numerical modelling of the contrast kinetics.

Keywords Carotid artery disease . Magnetic resonance angiography . Contrast enhancement - Dynamic . Fourier analysis
Abbreviations 3D: three- dimensional $\cdot$ CNR: contrast-to-noise ratio $\cdot$ MR: magnetic resonance · MRA: MR angiography · ROI: region of interest

\section{Introduction}

In carotid artery disease contrast-enhanced magnetic resonance (MR) angiography has become a noninvasive imaging alternative for catheter angiography [1-3]. The major task of carotid MRA is to assess carotid artery stenosis. Severe carotid stenosis $(70-99 \%$ according to NASCET criteria) is a major risk factor in ischaemic stroke, causing about $20 \%$ of cases [4]. Surgical endarterectomy and transluminal angioplasty make it a treatable risk factor $[5,6]$.
According to two current meta-analyses, gadoliniumenhanced carotid MRA has a high sensitivity of about $94 \%$ and a high specificity of about $92 \%$ for the diagnosis of severe carotid artery stenosis [7, 8]. Both meta-analyses treated gadolinium-enhanced carotid MRA as one entity, although this entity comprises several competitive methods. The three major methods are bolus-timed MRA, fluoroscopically triggered MRA and temporally resolved MRA. These MRA methods have similar diagnostic accuracy for the diagnosis of severe carotid stenosis; thus, they may be equally applied and further developed [7, 8]. 
This study investigated the image quality and contrast kinetics of bolus-timed carotid MRA, which is the traditional method. The observations in a patient collective with a relatively early acquisition of the contrast-enhanced MRA sequence are reported. Such short timing was applied to minimise venous enhancement while obtaining sufficient arterial enhancement. To explain the findings, bolustimed carotid MRA was modelled by Fourier-based numerical simulations that utilise the dynamic contrast information of the bolus time series.

\section{Materials and methods}

\section{Patients}

This study includes 100 prospectively collected carotid MRAs from 100 different consecutive patients with suspected or known carotid stenosis, thus representing a random sample from a high-risk patient collective. Seven additional carotid MRAs were excluded from the study: six were follow-up MRAs in the same patients and one MRA had major motion artefacts. These MRAs were replaced by consecutive MRAs to obtain the planned number of 100 MRAs. Within the study group the male-to-female ratio was 70:30, ages ranged from 44 to 86 years (median 70 years) and body weights ranged from 47 to $108 \mathrm{~kg}$ (median $78 \mathrm{~kg}$ ). The local ethics committee waived ethical approval on this study of image quality. Informed patient consent for MRA had been obtained. All MRA sequences were acquired solely for clinical reasons.

\section{Bolus-timed carotid MRA}

The carotid MRA were performed using 1.5-T magnets (Magnetom Symphony and Sonata, Siemens, Germany) with a phased-array neck coil. The patient was placed headfirst in the magnet. After multiplanar TruFISP localisers, the individual vein-to-carotid circulation time was measured by using a 2-ml gadolinium DTPA test bolus (gadopentetate dimeglumine, Magnevist, Schering, Germany). With an MR injector (Spectris Medrad, Indianola, USA) this test bolus was injected at a flow of $3 \mathrm{ml} / \mathrm{s}$ into an antecubital vein, followed by a $25-\mathrm{ml}$ saline flush at the same flow rate. Simultaneously, a time series of 40 transverse two-dimensional MR images near the carotid bifurcation was started with one image obtained per second (FLASH 2D, TR $1,000 \mathrm{~ms}$, TE $1.59 \mathrm{~ms}$, flip angle $8^{\circ}$, FOV $256 \times 256$ pixels, isotropic voxel size $1.37 \times 1.37 \mathrm{~mm}$ ). The MR technician placed regions of interest (ROIs) in the carotids of this bolus time series and read the arterial timeto-peak from the resulting time-signal curves. Then the supra-aortic arteries were imaged from the aortic arch to the circle of Willis with a breath-hold three-dimensional (3D) gradient echo sequence, resulting in 72 adjacent coronal slices (FLASH 3D, TR $3.54 \mathrm{~ms}$, TE $1.39 \mathrm{~ms}$, flip angle $25^{\circ}$, FOV $320 \times 208$ pixels, isotropic voxel size $1.1 \times 1.1 \times$ $1.1 \mathrm{~mm}$, acquisition time $21 \mathrm{~s}$ ). This 3D sequence was used with sequential $k$-space ordering and multidimensional 6/8 partial-Fourier acquisition in phase and slice encoding directions. According to the sequence parameter menu this 3D-MRA sequence reaches its $k$-space centre after $8.3 \mathrm{~s}$, which is slightly more than one third of the total acquisition time. First, such a 3D sequence was measured unenhanced. Then $0.2 \mathrm{ml} / \mathrm{kg}$ of gadolinium DPTA was intravenously injected and flushed with $25 \mathrm{ml}$ of saline, both at a flow of $3 \mathrm{ml} / \mathrm{s}$. A subsequent bolus-timed, gadolinium-enhanced 3D sequence was started, so that the central $k$-space acquisition coincided with the measured arterial time-to-peak from the bolus time series, using the following simple timing formula with $T_{\text {delta }}=0 \mathrm{~s}$ :

$T_{\text {start }}=T_{\text {art-peak }}-T_{k-\text { centre }}+T_{\text {delta }} \quad$ (timing formula)

with

$T_{\text {start }} \quad$ starting time of the $3 \mathrm{D}$ sequence after beginning the gadolinium injection

$T_{\text {art-peak }}$ individual arterial time-to-peak, as determined by the test bolus series

$T_{k \text {-centre }}$ time when the 3D sequence reaches its $k$-space centre, here rounded to $8 \mathrm{~s}$

$T_{\text {delta }} \quad$ delta time, for adding or subtracting any further timing factors

Usually, it is recommended to let $T_{\text {delta }}$ account for the injection time of the MRA gadolinium bolus $[9,10]$. At a constant flow rate of $3 \mathrm{ml} / \mathrm{s}$, the test bolus (2-ml volume) reaches its temporal centre after $0.3 \mathrm{~s}$, while the MRA bolus (about 15-ml volume) reaches its temporal centre after about $2.5 \mathrm{~s}$. Thus, for optimal arterial enhancement it would be necessary to add the difference of $T_{\text {delta }}=2.5-0.3=2.2 \mathrm{~s}$ to the starting time. However, the later the gadolinium-enhanced $3 \mathrm{D}$ sequence starts, the higher the risk of venous enhancement because of gadolinium that returns from the cranial circulation. In the study group $T_{\text {delta }}$ was set to $0 \mathrm{~s}$ as a compromise between sufficient arterial contrast and little venous contamination. After this bolus-timed acquisition of the gadoliniumenhanced 3D-MRA images the unenhanced 3D-MRA images were subtracted to suppress the background MR signal from surrounding neck tissue. From this subtracted 3D-MRA targeted maximum intensity projections (MIP) were derived to selectively depict the left and right supra-aortic arteries. Radiological diagnosis was based on the MIP images and on the original 3D images. The MRA images were stored in the PACS archive in the original DICOM format.

\section{Programs for postprocessing}

For this study the MR images were converted from the DICOM format to the ANALYZE format by using the free 
medical image viewer MRIcro (MRIcro 1.40, http://www. mricro.com). This program was also used for viewing images and drawing ROIs. Further postprocessing was done with proprietary programs written in $\mathrm{C}++6.0$ (Microsoft Corporation). Some of these programs utilised the free software FFTW (FFTW 3.1.2., http://www.fftw. org) for converting MR images to their spatial frequency domain ( $k$-space) and vice versa by forward and backward Fourier transform [11].

\section{ROI measurements}

Different ROI measurements were performed for image analysis as described below. To avoid interobserver variability, all ROIs were placed by the same author. This is consistent with the methods of several other studies [12-14]. Additionally this corresponds to clinical practice, where ROI measurements are usually performed by one radiologist.

\section{Analysis of test bolus time series}

In each individual test bolus series, ROIs were manually placed at the carotid arteries and the internal jugular veins. From corresponding arterial and venous time-signal curves different timing parameters were determined, as defined by Table 1 in conjunction with Fig. 1. These included the arterial time-to-peak ( $\left.T_{\text {art-peak }}\right)$ and other arterial, venous and mixed arteriovenous circulation times.

\section{Analysis of carotid MRA}

The angiographic enhancement of the carotid MRA (Fig. 2) was quantified by arterial and venous contrast-tonoise ratios (CNR). From the subtracted 3D-MRA a transverse plane was reformatted at the level of the test bolus sequence. On both sides of the neck ROIs were placed in the carotid arteries, internal jugular veins, neck muscles and in the air outside the neck. The arterial CNR, venous $\mathrm{CNR}$ and venous contamination were determined as follows:

$$
\begin{array}{ll}
\mathrm{CNR}_{\text {arterial }}=\left(\mathrm{SI}_{\text {carotids }}-\mathrm{SI}_{\text {muscle }}\right) / \mathrm{SD}_{\text {air }} & (\text { arterial CNR }) \\
\mathrm{CNR}_{\text {venous }}=\left(\mathrm{SI}_{\text {veins }}-\mathrm{SI}_{\text {muscle }}\right) / \mathrm{SD}_{\text {air }} & (\text { venous CNR })
\end{array}
$$$$
\text { Contam } \mathrm{venous}_{\mathrm{v}}=\mathrm{SI}_{\mathrm{veins}} / \mathrm{SI}_{\text {carotids }} \quad \text { (venous contamination) }
$$

with

$\mathrm{SI}_{\text {carotids }}$ mean signal intensity in the carotids

$\mathrm{SI}_{\text {veins }}$ mean signal intensity in the internal jugular veins

$\mathrm{SI}_{\text {muscle }}$ mean signal intensity in the neck muscles

$\mathrm{SD}_{\text {air }} \quad$ standard deviation of voxel intensities in the air

The amount of venous contamination was given as a percentage.

\section{Modelling of bolus-timed carotid MRA}

To explain the findings in the study group, bolus-timed carotid MRAs were modelled with different starting times for the 3D sequence in each individual. This modelling was solely based on clinical MRA images. No additional gadolinium was applied for this study. The modelling of bolus-timed MRA includes firstly, modelling the MRA bolus time series, and secondly, modelling the carotid MRA.

\section{Modelling of MRA bolus time series}

The test bolus had a 2-ml volume and was injected at a flow of $3 \mathrm{ml} / \mathrm{s}$ in less than $1 \mathrm{~s}$. Within the study group the MRA

\begin{tabular}{|c|c|c|c|c|}
\hline Timing parameter & Mean $(95 \% \mathrm{CI})(\mathrm{s})$ & Time interval $^{\mathrm{a}}$ & Symbol & Calculation \\
\hline Arterial time-to-begin & $13.6(8.5$ to 18.7$)$ & 0 to $\mathrm{A}$ & $T_{\mathrm{A}}$ & \\
\hline Arterial time-to-peak ( $\left.T_{\text {art-peak }}\right)$ & $19.4(12.0$ to 26.9$)$ & 0 to $\mathrm{B}$ & $T_{\mathrm{B}}$ & \\
\hline Arterial time-to-end & $29.2(18.8$ to 39.6$)$ & 0 to $\mathrm{C}$ & $T_{\mathrm{C}}$ & \\
\hline Venous time-to-begin & $21.8(14.1$ to 29.5$)$ & 0 to $\mathrm{D}$ & $T_{\mathrm{D}}$ & \\
\hline Venous time-to-peak & 29.5 (20.0 to 38.9$)$ & 0 to $\mathrm{E}$ & $T_{\mathrm{E}}$ & \\
\hline Arterial test bolus duration & $15.6(9.1$ to 22.1$)$ & A to $\mathrm{C}$ & $T_{\mathrm{F}}$ & $=T_{\mathrm{C}}-T_{\mathrm{A}}$ \\
\hline Pure arterial time-before-peak & $5.8(2.6$ to 9.1$)$ & $\mathrm{A}$ to $\mathrm{B}$ & $T_{\mathrm{G}}$ & $=T_{\mathrm{B}}-T_{\mathrm{A}}$ \\
\hline Pure arterial time-after-peak & $2.4(-0.5$ to 6.2$)$ & B to $\mathrm{D}$ & $T_{\mathrm{H}}$ & $=T_{\mathrm{D}}-T_{\mathrm{B}}$ \\
\hline Arteriovenous time-to-begin & $8.2(4.4$ to 12.0$)$ & A to $\mathrm{D}$ & $T_{\mathrm{I}}$ & $=T_{\mathrm{D}}-T_{\mathrm{A}}$ \\
\hline Arteriovenous time-to-peak & $10.0(6.1$ to 14.0$)$ & $\mathrm{B}$ to $\mathrm{E}$ & $T_{\mathrm{J}}$ & $=T_{\mathrm{E}}-T_{\mathrm{B}}$ \\
\hline
\end{tabular}

Table 1 Contrast dynamics of the test bolus in the study group

${ }^{a}$ The timing parameters correspond to the symbols $\mathrm{A}$ to $\mathrm{E}$ in Fig. 1. The intravenous injection of gadolinium started at $0 \mathrm{~s}$ 


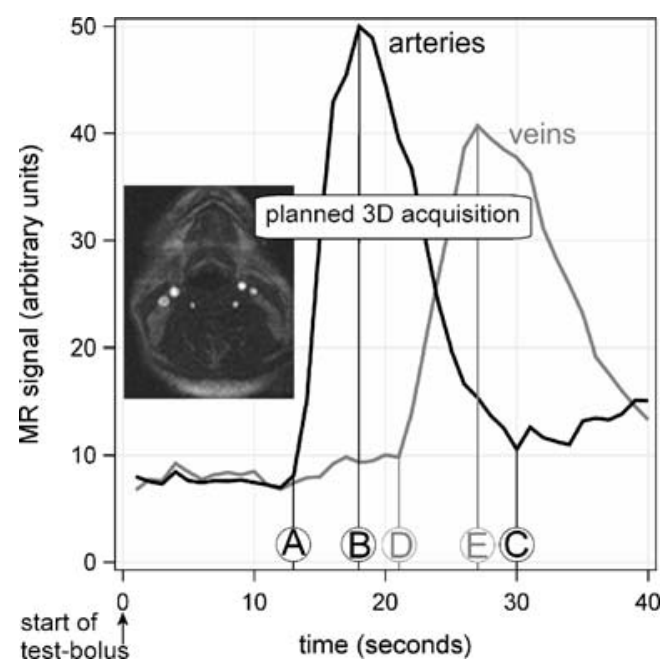

Fig. 1 Illustrative test bolus. Arterial and venous time-signal curves of the test bolus in a 68-year-old man. The test bolus reached the carotids at $13 \mathrm{~s}$ ( $A$ arterial time-to-begin), peaked with its MR signal at $T_{\text {art-peak }}=18 \mathrm{~s} \mathrm{(} B$ arterial time-to-peak $)$ and had passed the carotids at approximately $30 \mathrm{~s}$ ( $C$ arterial time-to-end). Marked enhancement of the internal jugular veins started at $21 \mathrm{~s}$ ( $D$ venous time-to-begin) and peaked at $27 \mathrm{~s}$ ( $E$ venous time-to-peak)

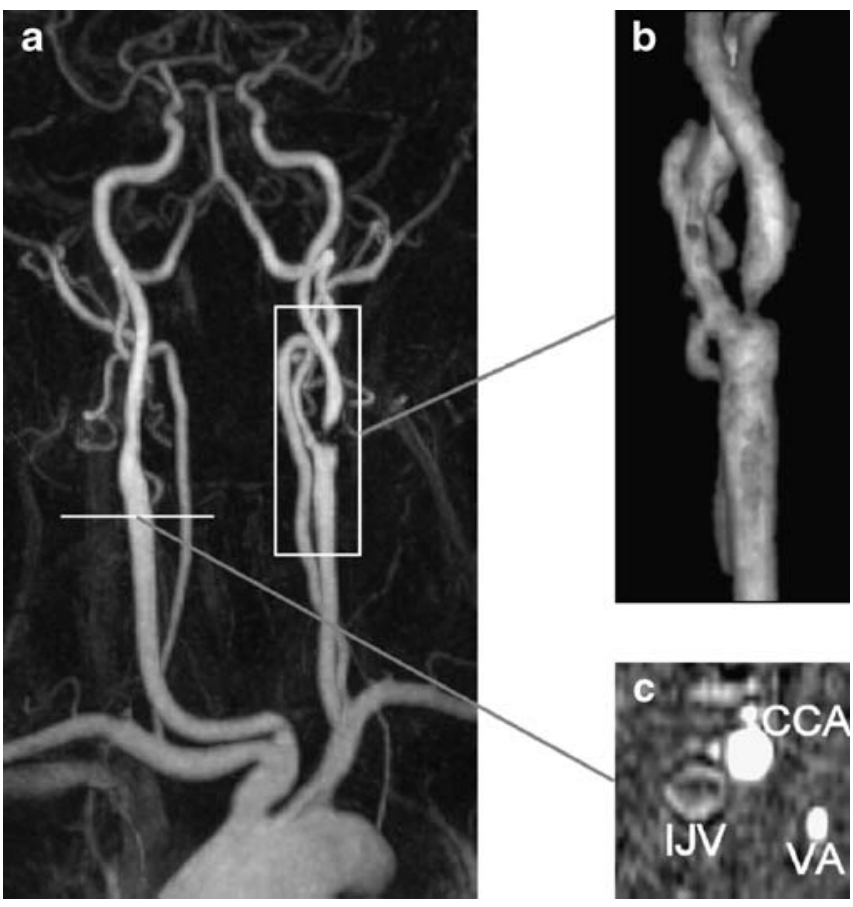

Fig. 2 Illustrative carotid MRA. Carotid MRA in the illustrative case: a coronal maximum intensity projection; b targeted volume rendering of an $80 \%$ stenosis of the proximal left internal carotid artery; c transverse plane of the 3D-MRA showing the right common carotid artery $(C C A)$, vertebral artery $(V A)$ and internal jugular vein $(I J V)$. The arteries are well enhanced. The IJV shows a ringing bolus for the subsequent gadolinium-enhanced 3D-MRA had a volume of about $15 \mathrm{ml}$ and was injected in about $5 \mathrm{~s}$. In all 100 cases the MRA bolus was modelled by superimposing temporally shifted time-signal curves of the test bolus as illustrated in Fig. 3. In detail, the MRA bolus was modelled as a 40 -s time series, as follows. The first 10 images of the test bolus time series were averaged to give an unenhanced mask image, similar to the mask image in digital subtraction angiography (DSA). This mask image was subtracted from the test bolus time series, similar to generating a series of DSA subtraction images. Such subtraction images retain the gadolinium-associated signal intensities, while the unenhanced background signal is subtracted. Several of these subtracted time series images were temporally shifted and superimposed to simulate the intravenous injection of $15 \mathrm{ml}$ gadolinium within $5 \mathrm{~s}$. Afterwards, the mentioned mask image was added again, so that the modelled MRA bolus time series also contained background signal. In these modelled MRA bolus time series the arterial and venous time-signal curves were calculated with the same ROIs as in the test bolus series. This was possible because these ROIs had been stored by the program MRIcro for such further applications.

\section{Modelling of carotid MRA}

The modelled MRA bolus time series were also used for modelling the arteriovenous contrast dynamics of carotid MRA with different starting times by varying $T_{\text {delta }}$ in the

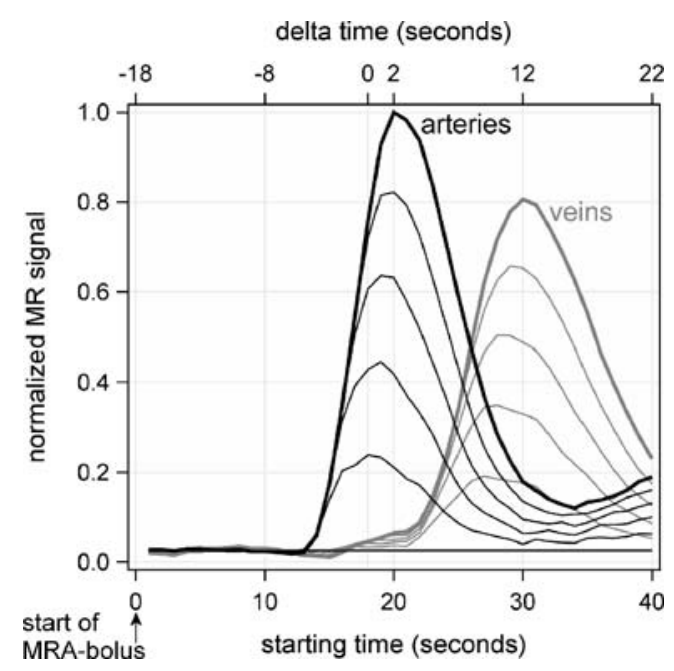

Fig. 3 Modelling of MRA bolus kinetics. The gadolinium bolus for the 3D-MRA was of larger volume and was injected over a longer period than the test bolus. Five time-shifted test bolus time-signal curves (thin lines, normalised to $3 \mathrm{ml}$ of gadolinium DTPA) were superimposed to model the arterial and venous time-signal curves of a 5-s-long MRA bolus with $15 \mathrm{ml}$ of gadolinium DTPA (upper thick lines). The resulting arterial curve peaked at $20 \mathrm{~s}$ and thus $2 \mathrm{~s}$ later than with the shorter test bolus. The maximum MR signal on the $y$ axis has been normalised to 1 
timing formula. For this purpose a sequential acquisition with a 21 -s duration and $81 \%$ partial-Fourier (i.e. slightly more than $6 / 8=75 \%$ ) was modelled, as illustrated in Fig. 4 [11]. This modelled sequence reaches its $k$-space centre after $T_{k \text {-centre }}=8 \mathrm{~s}$, which is approximately similar to the $8.3 \mathrm{~s}$ of the real MRA sequence that was applied in the patients. The arterial CNR, the venous CNR and the amount of venous contamination of the modelled carotid MRA were calculated with the same ROIs as in the MRA bolus time series. This modelling of carotid MRA is restricted to the two-dimensional plane of the bolus time series. However, the signal intensities of the real 3D-MRA were also evaluated in this transverse plane, so that the two-dimensional modelling of this MRA plane is considered sufficient for explaining the arteriovenous contrast dynamics of the 3D-MRA in this study.

\section{Results}

\section{Illustrative case}

Figure 1 presents the arterial and venous time-signal curves of the gadolinium test bolus in a 68 -year-old man with an $80 \%$ stenosis of the proximal left internal carotid artery. The 2-ml test bolus had been intravenously injected in less than $1 \mathrm{~s}$. Because of intravascular dispersion the bolus is prolonged to 17 -s duration (A to $\mathrm{C}$ ) while flowing from the antecubital vein through the cardiopulmonary circulation to the carotids. The peak arterial signal (B) was reached at $T_{\text {art-peak }}=18 \mathrm{~s}$ and the time window of pure arterial enhancement (A to D) was $8 \mathrm{~s}$, which is representative of the study group. Marked enhancement of the internal jugular veins started at $21 \mathrm{~s}$ (D), i.e. $3 \mathrm{~s}$ after the arterial peak. These contrast kinetics illustrate that the optimal timing of carotid MRA depends on a few seconds. The subsequent gadolinium-enhanced 3D-MRA was planned to start at $T_{\text {start }}=10 \mathrm{~s}$, i.e. with $T_{\text {delta }}=0 \mathrm{~s}$, according to the timing formula. Thus, not only the mixed arterialvenous signal after the arterial peak (after B), but also the $5 \mathrm{~s}$ of pure arterial signal before the arterial peak (from A to B), was used for angiographic imaging.

Figure 2 shows a coronal MIP image of the subsequent 3D-MRA. The cervical arteries are well demonstrated with a high arterial CNR (contrast-to-noise ratio) of 61.8. Although the gadolinium bolus was well timed, there is already venous enhancement with a venous CNR of 3.4 in the internal jugular veins, causing slight venous contamination of $8.7 \%$ on the MRA image. In Fig. $2 \mathrm{c}$ the arteries are homogeneously enhanced, whereas the internal jugular vein (IJV) shows a typical ringing, as if it had been spatially high-pass filtered. a
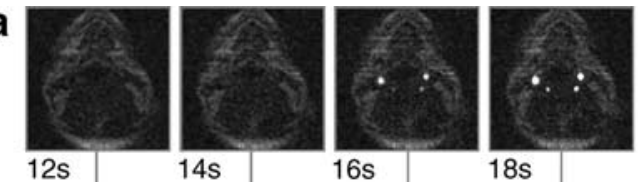

b
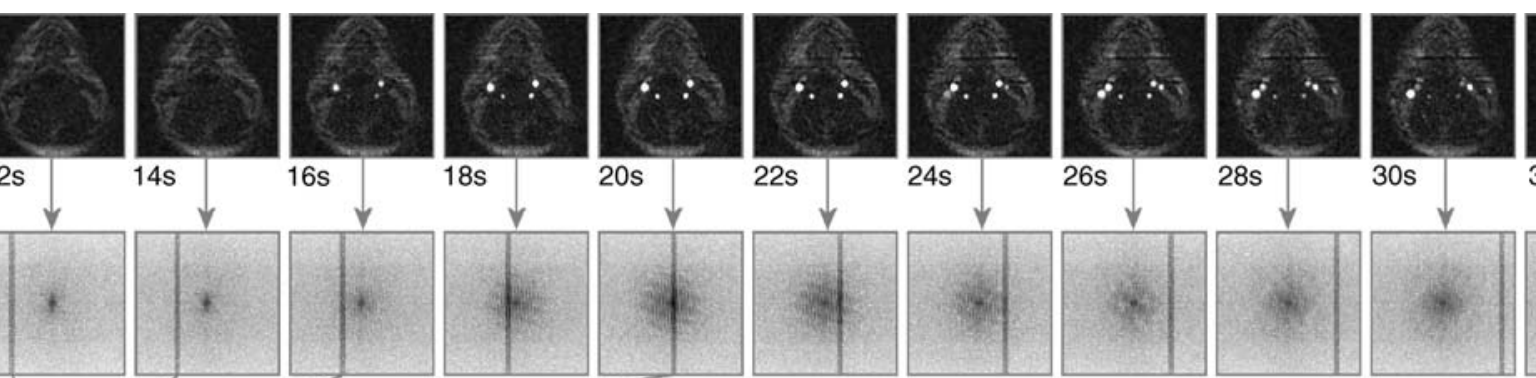

$32 s$
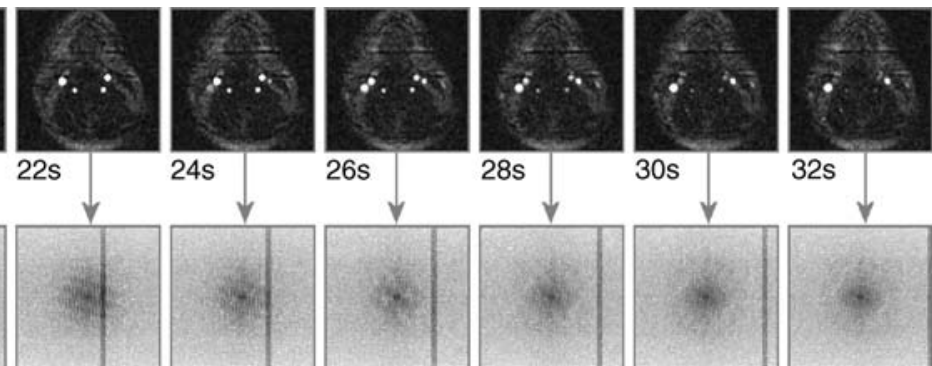

e
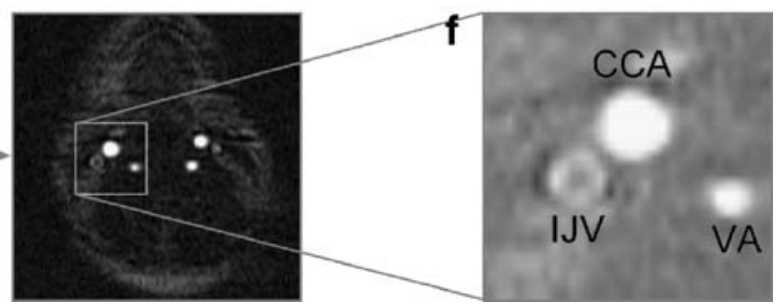

Fig. 4 Modelling of gadolinium-enhanced MRA acquisition in the illustrative case, if it had been started at $T_{\text {start }}=12 \mathrm{~s}$ to achieve the highest possible arterial contrast: a modelled transverse 2D MRA images at different times after the beginning of intravenous injection of gadolinium; b by fast Fourier transform the MR images are transformed to their spatial frequency domain ( $k$-space); c modelling a 21-s MRA sequence with slightly more than $6 / 8$ partial-Fourier acquisition (with $81 \%$ instead of $75 \% k$-space coverage) by linking the dark-marked $k$-space segments in $\mathbf{b}$ from different times and

different spatial frequencies; d postprocessed $k$-space image with Hermitian symmetry; e inverse Fourier transform of $\mathbf{d}$ gives the final MRA image as modelled with the 21-s sequence; $f$ the common carotid artery $(C C A)$ and the vertebral artery $(V A)$ are homogeneously contrasted with their highest possible arterial signal. However, the modelled MRA image already shows some venous enhancement in the internal jugular vein $(I J V)$ with a typical ringing, similar to the observed ringing in Fig. 2c 
Observations in the study group

Table 1 summarises the test bolus dynamics in the study group. The arterial enhancement peak is considered optimal for acquisition of the central $k$-space. Therefore, the arterial time-to-peak ( $\left.T_{\text {art-peak }}\right)$ was used for bolus timing. In the study group the average $T_{\text {art-peak }}$ was $19.4 \mathrm{~s}$. However, this $T_{\text {art-peak }}$ varied considerably among patients $(95 \%$ confidence interval $12.0-26.9 \mathrm{~s})$, indicating the need for individual timing of the gadoliniumenhanced 3D-MRA sequence. In the study group the arterial test bolus lasted on average $15 \mathrm{~s}$. Of this, the initial time window of pure arterial enhancement was on average $8 \mathrm{~s}$, before the venous enhancement started. Of note, the venous enhancement started on average only $2.4 \mathrm{~s}$ after the arterial peak, and peaked itself $10 \mathrm{~s}$ later. This early venous enhancement explains why even an optimally timed 3D-MRA sequence may show some venous contamination.

Table 2 summarises the image quality parameters of the 3D-MRA in the study group. The arterial contrast-to-noise ratio (CNR) was generally very high, so that the cervical arteries were well depicted on angiographic maximum intensity projection (MIP) images. In most cases the venous enhancement and thus the venous contamination was small. A few cases showed venous contamination of up to $16.3 \%$. However, this relatively small amount of venous contamination did not hinder visualisation of the arteries, because the arterial MR signals were still much higher than the venous MR signals. All 100 MRAs were of diagnostic quality, and all arteries were homogeneously contrasted without ringing signs. In the cases with highest venous enhancement the veins typically showed a marginal ringing enhancement (Fig. 2c). As mentioned in Material and methods, only one MRA had been excluded from the study because of major motion artefacts, but not because of insufficient arterial contrast or excessive venous enhancement.

Table 2 Image quality of the 3D-MRAs in the study group

\begin{tabular}{lccc}
\hline & $\begin{array}{l}\text { Arterial } \\
\mathrm{CNR}^{\mathrm{a}}\end{array}$ & $\begin{array}{l}\text { Venous } \\
\mathrm{CNR}^{\mathrm{a}}\end{array}$ & $\begin{array}{l}\text { Venous } \\
\text { contamination }(\%)^{\mathrm{a}}\end{array}$ \\
\hline Mean & 53.0 & 1.2 & 6.4 \\
SD & 12.8 & 1.9 & 2.7 \\
Minimum & 31.9 & -1.1 & 2.5 \\
5th percentile & 34.9 & -0.6 & 3.4 \\
Median & 51.7 & 0.7 & 5.6 \\
95th percentile & 74.6 & 5.3 & 11.8 \\
Maximum & 98.1 & 10.6 & 16.3 \\
\hline
\end{tabular}

${ }^{\mathrm{a}} \mathrm{CNR}$ and venous contamination are defined in Material and methods
Modelling of bolus-timed carotid MRA

in the illustrative case

The time series of the short test bolus ( $2 \mathrm{ml}$ of gadolinium DTPA injected in less than $1 \mathrm{~s}$ ) was used to model the contrast kinetics of the longer MRA bolus (about $15 \mathrm{ml}$ of gadolinium DTPA injected in $5 \mathrm{~s}$ ). Figure 3 shows this modelling in the illustrative case. The modelled arterial signal of the longer MRA bolus peaked at $20 \mathrm{~s}$, and thus $2 \mathrm{~s}$ later than in the test bolus $\left(T_{\text {art-peak }}=18 \mathrm{~s}\right)$. According to this modelled MRA bolus the carotid MRA should be started with $T_{\text {delta }}=+2 \mathrm{~s}$ to achieve the highest possible arterial contrast. With the timing formula the optimal starting time would then be $T_{\text {start }}=18-8+2=12 \mathrm{~s}$ after the beginning of the intravenous injection of gadolinium.

Figure 4 shows the modelling of bolus-timed carotid MRA with this starting time in the illustrative case. The modelled arteries are homogeneously and well contrasted. However, the modelled internal jugular vein is also somewhat enhanced and shows a typical ringing in Fig. $4 \mathrm{f}$ that was also observed in the real measured carotid MRA in Fig. 2c. This venous ringing occurs when the vein is initially unenhanced, but fills with gadolinium during acquisition of the final high-frequency components of the peripheral $k$-space. This can be seen in Fig. $4 \mathrm{a}$ and $\mathrm{b}$ between 24 and $32 \mathrm{~s}$ after the beginning of the intravenous injection of gadolinium. Thus, in the MRA image this venous enhancement can only contribute to high-frequency waves. This is why the internal jugular vein in the measured (Fig. 2c) and in the modelled (Fig. 4f) carotid MRA seems to have been spatially high-pass filtered. The primary bolus images in Fig. 4a do not show this venous ringing because of a much shorter acquisition time per image.

\section{Modelling of different MRA starting times}

The modelling of carotid MRA with different starting times is shown in Fig. 5a for the illustrative patient and in Fig. 5b for the study group.

In the illustrative case (Fig. 5a) the MRA sequence had originally been planned to start at $T_{\text {start }}=18-8=10 \mathrm{~s}$ according to the timing formula, i.e. $T_{\text {delta }}=0 \mathrm{~s}$. With this early MRA timing the modelled arterial MR signal is about $84 \%$ of its theoretical maximum, and the modelled venous MR signal is $3.9 \%$ of the maximal arterial signal. This results in a modelled venous contamination of $4.7 \%$. However, with this $T_{\text {delta }}=0 \mathrm{~s}$ the arterial signal does not reach its theoretical maximum. This overall maximum is reached $2 \mathrm{~s}$ later, which is consistent with Fig. 3. When considering these $2 \mathrm{~s}$, the MRA should be started with $T_{\text {delta }}=2 \mathrm{~s}$, i.e. at $T_{\text {start }}=18-8+2=12 \mathrm{~s}$ after the beginning of the gadolinium injection. In this case the $k$-space centre is acquired while the arterial gadolinium concentration of the MRA bolus is highest. However, because of the early 


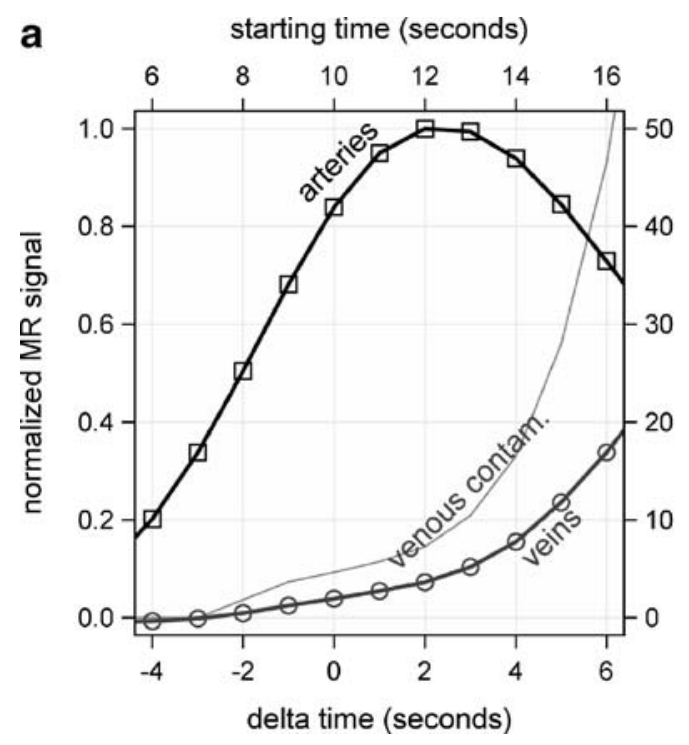

Fig. 5 Modelling of bolus-timed MRA showing the arterial and venous MR signals and the percentage of venous contamination of the modelled carotid MRA at different starting times a in the illustrative case, and $\mathbf{b}$ in the whole study groups after coherent

venous return of gadolinium the resulting venous contamination is then already $7.2 \%$. If the MRA acquisition is further delayed by an additional $2 \mathrm{~s}$, the MRA would start at $T_{\text {delta }}=4 \mathrm{~s}$, i.e. at $T_{\text {start }}=18-8+4=14 \mathrm{~s}$ after the beginning of the intravenous injection of gadolinium. According to the model, the venous contamination is then already about $17 \%$ (Fig. 5a). The real acquired 3D-MRA in the illustrative case showed venous contamination of $8.7 \%$ (Fig. 2). Perhaps this 3D-MRA had been acquired with a $T_{\text {delta }}$ of about $2 \mathrm{~s}$, and thus slightly later than planned.

Both parts of Fig. 5 are relatively similar, which indicates that the considerations about different MRA starting times in the illustrative patient can be transferred to the study group. In summary this modelling of contrast kinetics shows that the timing of gadolinium-enhanced carotid MRA is sensitive to a few seconds. A relatively short timing with $T_{\text {delta }}=0 \mathrm{~s}$ is a compromise that provides sufficient arterial contrast with little venous contamination.

\section{Discussion}

In carotid artery disease contrast-enhanced magnetic resonance (MR) angiography is a well-established noninvasive imaging method [1-3]. Several methods exist to achieve high arterial contrast with little venous contamination, which much depends on contrast kinetics. The gadolinium chelates are usually injected into an antecubital vein. Although being injected in less than $1 \mathrm{~s}$, the test bolus duration is prolonged to about $15 \mathrm{~s}(95 \%$ CI 9-22 s) while flowing through the cardiopulmonary circulation to the carotids. Because of this bolus dispersion there is generally no risk that the time window of carotid enhancement is too

\section{b delta time (seconds)}

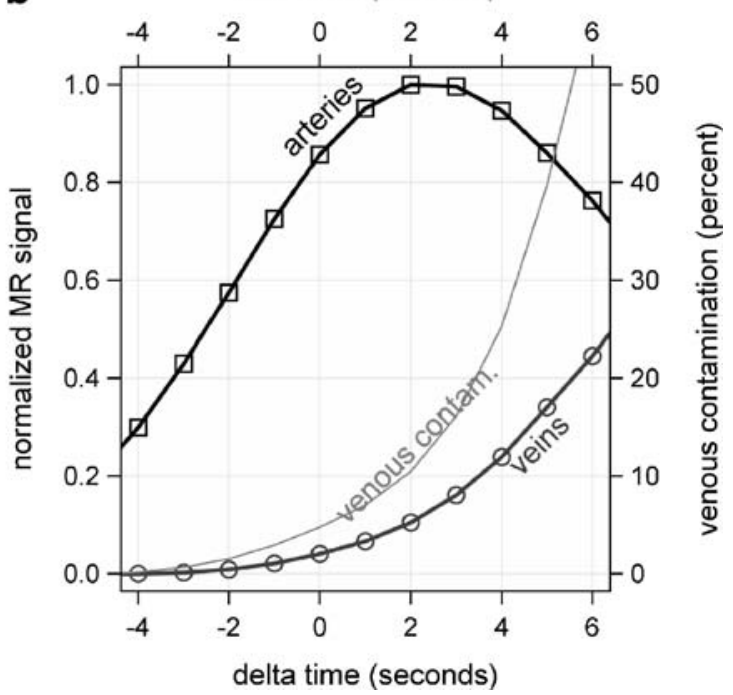

averaging of the individual $T_{\text {delta. }}$ The MR signals are normalised to 1 for the maximal possible arterial MR signal. With $T_{\text {delta }}=0 \mathrm{~s}$ the venous contamination is relatively small and the arterial signal is sufficiently high. Further details are given in Results

short, even if the MRA bolus is injected at a high flow of about $3 \mathrm{ml} / \mathrm{s}$ in a few seconds. The major temporal limitation of this arterial acquisition window originates from venous enhancement because of gadolinium that returns from the cranial circulation: the pure arterial time window lasted on average only $8 \mathrm{~s}$ in the study group, which is quite short.

Because of the fast cervicocranial circulation the timing of carotid MRA depends on a few seconds, as indicated by this study and by others [9, 10]. Basically this is well known. However, this study adds considerations about venous contamination of the MRA images. Usually it is recommended to let the acquisition of the central $k$-space coincide with the arterial bolus peak to achieve the highest arterial MR signal $[9,10]$. However, in carotid MRA this general rule does not account for venous contamination because of early venous return from the cranial circulation. In the presented study the carotid MRA was started relatively early with $T_{\text {delta }}=0 \mathrm{~s}$ according to the timing formula. This does not account for the fact that the MRA bolus had more volume and thus needed about $2 \mathrm{~s}$ longer to reach its peak than the test bolus. According the Fourierbased modelling the carotid MRAs thus achieved only about $84 \%$ of the maximal possible arterial contrast. However, with this relatively early acquisition of the 3DMRA the observed venous contamination was on average small, so that $T_{\text {delta }}=0 \mathrm{~s}$ offers a clinically useful compromise between sufficient arterial contrast and little venous contamination.

All studied carotid MRA showed a high arterial contrastto-noise ratio (CNR) and homogeneously enhanced arteries without ringing. This indicates that the 3D-MRA had not been started too early, and that the central $k$-space of the 
3D-MRA was acquired sufficiently near to the arterial enhancement peak. When the internal jugular veins were slightly enhanced, they typically showed a ringing that can be explained well by Fourier-based modelling (Figs. 2c and 4f). Generally the venous contamination was sufficiently small and did not hinder visualization of the well-enhanced arteries.

This study did not intend to compare bolus-timed MRA with other competitive MRA techniques. In gadoliniumenhanced carotid MRA there seems to be no simple rule that predicts an optimum in the complex relation between diagnostic accuracy and different MRA parameter settings. Such parameters include the type of MRA timing, sequence duration, $k$-space acquisition order, spatial resolution, gadolinium bolus details, and others. For example, several studies have been published about the sensitivity and specificity of carotid MRA in the diagnosis of severe carotid stenosis. These studies utilized a broad variety of different MRA parameter settings that are in clinical use. With all these different methods the diagnostic accuracy of carotid MRA was generally excellent, independent of the applied MRA settings. In this context, the results of bolustimed MRA, fluoroscopically triggered MRA and timeresolved MRA were all good. The traditional bolus-timed carotid MRA offers the same high diagnostic accuracy as the other MRA timing methods and should thus not be excluded from further technical developments.

The obtained carotid MRAs in the study group are observations and are as such not limited. However, the explanatory modelling of contrast kinetics is based on simulations, which are generally known to fit the reality not always exactly. A more exact approach would have been to study real contrast kinetics with several different starting times $T_{\text {start }}$ in each patient. According to the timing formula, the data for Fig. 5b could then be obtained by measuring 11 bolus time series in each patient, while varying $T_{\text {delta }}$ from -4 to $+6 \mathrm{~s}$ and injecting about $15 \mathrm{ml}$ gadolinium DTPA for each measurement. This would necessitate a total dose of $165 \mathrm{ml}$ gadolinium DTPA per patient. However, then the study would have an experimental instead of its observational character. For the presented observational study the numerical modelling of the gadolinium contrast kinetics is considered to be sufficient, because this modelling is solely used to explain the clinical findings with $T_{\text {delta }}=0 \mathrm{~s}$. A further study limitation is that the arteriovenous contrast kinetics is only modelled for a two-dimensional transverse plane of the neck. It would be desirable to measure and model the contrast kinetics not only with a two-dimensional but also with a three-dimensional sequence at similar spatiotemporal resolution. This might be achieved with parallel imaging and a very high acceleration factor.

Recently carotid MRA with parallel imaging has been reported with a sixfold acceleration factor [15]. Interestingly this study also used partial-Fourier acquisition and bolus timing, indicating that bolus-timed carotid MRA is not an outdated technology [15]. Such high-resolution carotid MRA could further help to diagnose carotid artery disease without radiation, and might thus aid in the framework of stroke prevention $[5,6,16]$. Bolus timing might remain part of such MRA techniques, when it is the aim to fully exploit the arterial contrast bolus with planned little venous contamination.

\section{Conclusion}

In bolus-timed carotid MRA a relatively short timing provides good arterial contrast with little venous contamination, which can be explained by Fourier-based numerical modelling of the contrast kinetics.

Open Access This article is distributed under the terms of the Creative Commons Attribution Noncommercial License which permits any noncommercial use, distribution, and reproduction in any medium, provided the original author(s) and source are credited.

\section{References}

1. The European Stroke Organisation (ESO) Executive Committee and the ESO Writing Committee (2008) Guidelines for management of ischaemic stroke and transient ischaemic attack. Cerebrovasc Dis 25:457-507
2. Qureshi AI, Alexandrov AV, Tegeler CH, Hobson RW, Baker JD, Hopkins LN (2007) Highlights of the guidelines for screening of extracranial carotid artery disease: a statement for healthcare professionals from the Multidisciplinary Practice Guidelines Committee of the American Society of Neuroimaging; cosponsored by the Society of Vascular and Interventional Neurology. J Endovasc Ther 14:469-474
3. Korteweg MA, Kerkhoff H, Bakker J, Elgersma OE (2008) Efficacy of patient selection strategies for carotid endarterectomy by contrast-enhanced MRA on a $1 \mathrm{~T}$ machine and duplex ultrasound in a regional hospital. Clin Radiol 63:174-183 
4. Grau AJ, Weimar C, Buggle F et al (2001) Risk factors, outcome, and treatment in subtypes of ischemic stroke: the German stroke data bank. Stroke 32:2559-2566

5. Sacco RL, Adams R, Albers G et al (2006) Guidelines for prevention of stroke in patients with ischemic stroke or transient ischemic attack: a statement for healthcare professionals from the American Heart Association/American Stroke Association Council on Stroke. Circulation 113:e409-e449

6. Goldstein LB, Adams R, Alberts MJ et al (2006) Primary prevention of ischemic stroke: a guideline from the American Heart Association/American Stroke Association Stroke Council. Circulation 113:e873-e923
7. Wardlaw JM, Chappell FM, Best JJ, Wartolowska K, Berry E (2006) Noninvasive imaging compared with intraarterial angiography in the diagnosis of symptomatic carotid stenosis: a metaanalysis. Lancet 367:1503-1512

8. Debrey SM, Yu H, Lynch JK et al (2008) Diagnostic accuracy of magnetic resonance angiography for internal carotid artery disease: a systematic review and meta-analysis. Stroke 39:2237-2248

9. Maki JH, Prince MR, Londy FJ, Chenevert TL (1996) The effects of time varying intravascular signal intensity and k-space acquisition order on three-dimensional MR angiography image quality. J Magn Reson Imaging 6:642-651

10. Zhang H, Maki JH, Prince MR (2007) 3D contrast-enhanced MR angiography. J Magn Reson Imaging 25:13-25

11. Frigo M, Johnson SG (2005) The design and implementation of FFTW3 Proc IEEE 93:216-231

12. Horiguchi J, Fukuda H, Yamamoto $\mathrm{H}$ et al (2007) The impact of motion artifacts on the reproducibility of repeated coronary artery calcium measurements. Eur Radiol 17:81-86
13. Werder R, Nanz D, Lutz AM et al (2007) Assessment of the abdominal aorta and its visceral branches by contrast-enhanced dynamic volumetric hepatic parallel magnetic resonance imaging: feasibility, reliability and accuracy. Eur Radiol 17:541-551

14. Willinek WA, Bayer T, Gieseke J et al (2007) High spatial resolution contrastenhanced MR angiography of the supraaortic arteries using the quadrature body coil at $3.0 \mathrm{~T}$ : a feasibility study. Eur Radiol 17:618-625

15. Lohan DG, Barkhordarian F, Saleh R et al (2007) MR angiography at $3 \mathrm{~T}$ for assessment of the external carotid artery system. AJR Am J Roentgenol 189:1088-1094

16. US Preventive Services Task Force (2007) Screening for carotid artery stenosis: US Preventive Services Task Force recommendation statement. Ann Intern Med 147:854-859 